\title{
Human Skin Color Simulator Using Active Illumination
}

\author{
Motonori Doi ${ }^{1}$, Akira Kimachi ${ }^{1}$, Shogo Nishi ${ }^{1}$, and Shoji Tominaga ${ }^{2}$ \\ ${ }^{1}$ Osaka Electro-Communication University, 18-8 Hatsu-cho, Neyagawa, \\ Osaka 572-8530, Japan \\ ${ }^{2}$ Chiba University, 1-33, Yayoi-cho, Inage-ku, Chiba 263-8522, Japan \\ \{doi,kima,s-nishi\}@isc.osakac.ac.jp, shoji@faculty.chiba-u.jp
}

\begin{abstract}
Skin color simulation is one of the most important topics. We propose a novel method to reproduce realistic skin color under different conditions by projecting active illumination to a real human skin surface. The active illumination is generated by a programmable light source, which can emit illuminant in high speed with any spectral distribution. The spectral reflectances of human skin with different concentrations of pigments in the skin are estimated based on the Kubelka-Munk theory. Then, the appearance of the skin surface under arbitrary illuminant is displayed by projecting the programmed light onto a real human skin surface. The experimental results show the feasibility of the proposed method.
\end{abstract}

Keywords: color simulation, skin color, programmable light source, spectral radiance.

\section{Introduction}

Color simulation of human skin is one of the most important topics. Skin color appearance is changed by illumination and the concentration of pigments in the skin. The surface layer of human skin is composed of two turbid layers of the epidermis and dermis. Each layer includes pigments of melanin and hemoglobin respectively. The surface spectral reflectance then depends on the concentration of the pigments. Production of realistic appearance of human skin under a variety of conditions is useful for many research fields including medical imaging, cosmetic development and computer graphics.

In the previous work [1] we described an algorithm for estimating surface-spectral reflectance of human skin using the Kubelka-Munk theory [2,3]. The skin color was then reproduced using the estimated spectral reflectance on a calibrated RGB color display. However, it was difficult to calibrate the color on the monitor correctly. The color matching between spectral data and RGB values on monitor is not simple problem. In this paper, we propose a direct approach to spectral reproduction of human skin under different conditions by using an active illumination system and a real human skin. Tominaga, et al. constructed a visual evaluation system for objects in a scene under arbitrary illuminants [4,5]. The system was a high-speed spectral imaging system using an active spectral illumination, where 3D object surfaces in a real scene can be observed by synthesizing illuminant with arbitrary spectral-power distribution. In this paper we produce realistic appearance of skin by projecting illuminant 
with a specific spectral-power distribution onto real skin surface. The surface appearance of human skin with curved texture can be produced directly under different pigment conditions, without using tristimulus calculation and RGB color conversion.

\section{Color Simulation by Active Illumination}

\subsection{Principles of Color Simulation}

We assume a simple model for the surface reflection. The observed object surface color is determined from the spectral distributions of light and the spectral reflectance of the surface. Fig. 1 shows the model of color simulation by active illumination. In this model, the observed spectral radiance $V_{\mathrm{o}}(\lambda)$ of the object with spectral reflectance $R_{\mathrm{o}}(\lambda)$ under the illumination with the spectral power distribution $E(\lambda)$ is described as the following equation,

$$
V_{\mathrm{o}}(\lambda)=R_{\mathrm{o}}(\lambda) E(\lambda)
$$

where $\lambda$ is the parameter of wavelength. The reproduction of the simulated color on the object is done by the illumination with the spectral power distribution $E^{\prime}(\lambda)$ to the object,

$$
E^{\prime}(\lambda)=E(\lambda) \frac{R_{\mathrm{S}}(\lambda)}{R_{\mathrm{o}}(\lambda)}
$$

where $R_{\mathrm{S}}(\lambda)$ is the simulated spectral reflectance of object. The observed spectral radiance $V_{\mathrm{S}}(\lambda)$ of the object with reflectance $R_{\mathrm{S}}(\lambda)$ under the illumination with the spectral power distribution $E(\lambda)$ is equal to the observed color of object with reflectance $R_{\mathrm{o}}(\lambda)$ under the illumination with the spectral power distribution $E^{\prime}(\lambda)$.
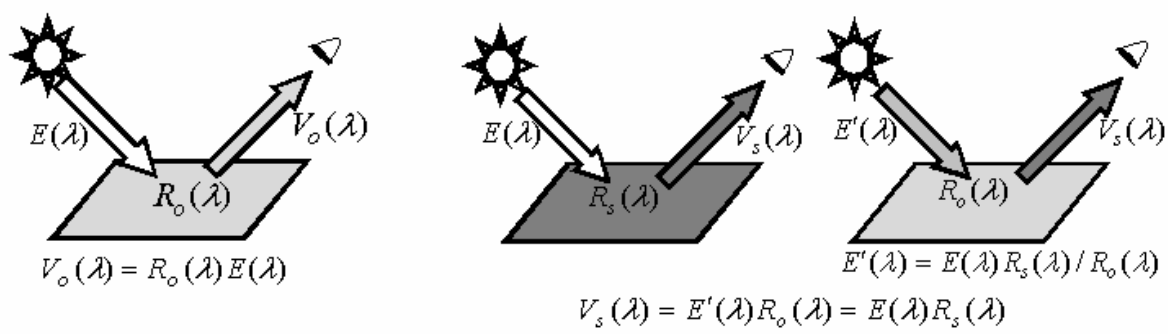

Fig. 1. Principles of color simulation by active illumination

\subsection{Programmable Light Source}

It is difficult to control the spectral power distribution of illumination by using conventional light source. We use a programmable light source (Optronic Laboratories, Inc. OL490) to control the illumination spectra. Fig. 2 shows the programmable light source. The light source can emit light with arbitrary spectral-power distribution. 


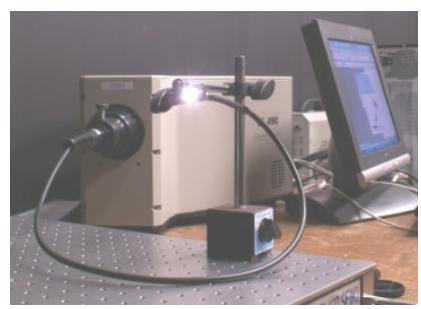

Fig. 2. Programmable light source

The programmable light source is composed of a xenon lamp source, a grating, a digital micro mirror device (DMD) chip, and a liquid light guide. The light beam of xenon is separated by the grating into its constituent wavelength. The intensity of the light on each wavelength is controlled by the DMD chip.

\section{Skin Color Estimation}

The skin color depends on some histological variables such as the concentration of pigments of melanin and hemoglobin. Our previous work [1] showed that the skin surface reflectance was controllable by the density parameters of melanin and hemoglobin. In our method, the human skin was modeled in the two turbid layers of the epidermis including melanin and dermis including hemoglobin as shown in Fig.3. In this model, the incident light penetrating the skin surface is absorbed and scattered in these two layers. The spectral reflectance and spectral transmittance of each layer was determined from density parameters by the Kubelka-Munk theory [2,3].

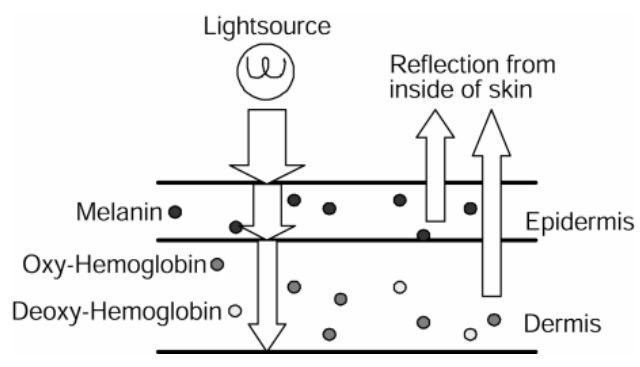

Fig. 3. Skin optics model

The body reflectance function of the skin surface $R_{\mathrm{b}}(\lambda)$ is described as a function of wavelength as

$$
R_{\mathrm{b}}(\lambda)=R_{\mathrm{e}}(\lambda)+\frac{T_{\mathrm{e}}(\lambda)^{2} R_{\mathrm{dt}}(\lambda)}{1-R_{\mathrm{e}}(\lambda) R_{\mathrm{dt}}(\lambda)},
$$


where $R_{\mathrm{e}}(\lambda)$ and $T_{\mathrm{e}}(\lambda)$ are, respectively, the spectral reflectance and transmittance of epidermis. The spectral reflectance $R_{\mathrm{dt}}(\lambda)$ represents the reflectance of dermis and the equation for $R_{\mathrm{dt}}(\lambda)$ is described as

$$
R_{\mathrm{dt}}(\lambda)=R_{\mathrm{d}}(\lambda)+\frac{T_{\mathrm{d}}(\lambda)^{2}}{1-R_{\mathrm{d}}(\lambda)},
$$

where $R_{\mathrm{d}}(\lambda)$ and $T_{\mathrm{d}}(\lambda)$ are the spectral reflectance and transmittance of the dermis, respectively. We assume that the reflectance of tissues under dermis is 1 .

The spectral reflectances and transmittances of the respective layers are given as follows:

Epidermis:

$$
\begin{aligned}
& R_{\mathrm{e}}(\lambda)=\frac{1}{a_{\mathrm{e}}(\lambda)+b_{\mathrm{e}}(\lambda) \operatorname{coth} b_{\mathrm{e}}(\lambda) S_{\mathrm{e}}(\lambda) D_{\mathrm{e}}} \\
& T_{\mathrm{e}}(\lambda)=\frac{b_{\mathrm{e}}(\lambda)}{a_{\mathrm{e}}(\lambda) \sinh b_{\mathrm{e}}(\lambda) S_{\mathrm{e}}(\lambda) D_{\mathrm{e}}+b_{\mathrm{e}}(\lambda) \operatorname{coth} b_{\mathrm{e}}(\lambda) S_{\mathrm{e}}(\lambda) D_{\mathrm{e}}} \\
& a_{\mathrm{e}}(\lambda)=\frac{S_{\mathrm{e}}(\lambda)+K_{\mathrm{e}}(\lambda)}{S_{\mathrm{e}}(\lambda)}, b_{\mathrm{e}}(\lambda)=\sqrt{a_{\mathrm{e}}(\lambda)^{2}-1}
\end{aligned}
$$

Dermis:

$$
\begin{aligned}
& R_{\mathrm{d}}(\lambda)=\frac{1}{a_{\mathrm{d}}(\lambda)+b_{\mathrm{d}}(\lambda) \operatorname{coth} b_{\mathrm{d}}(\lambda) S_{\mathrm{d}}(\lambda) D_{\mathrm{d}}} \\
& T_{\mathrm{d}}(\lambda)=\frac{b_{\mathrm{d}}(\lambda)}{a_{\mathrm{d}}(\lambda) \sinh b_{\mathrm{d}}(\lambda) S_{\mathrm{d}}(\lambda) D_{\mathrm{d}}+b_{\mathrm{d}}(\lambda) \operatorname{coth} b_{\mathrm{d}}(\lambda) S_{\mathrm{d}}(\lambda) D_{\mathrm{d}}} \\
& a_{\mathrm{d}}(\lambda)=\frac{S_{\mathrm{d}}(\lambda)+K_{\mathrm{d}}(\lambda)}{S_{\mathrm{d}}(\lambda)}, b_{\mathrm{d}}(\lambda)=\sqrt{a_{\mathrm{d}}(\lambda)^{2}-1}
\end{aligned}
$$

where $S_{\mathrm{e}}(\lambda)$ and $S_{\mathrm{d}}(\lambda)$ are spectral scattering coefficients in epidermis and dermis, respectively. The spectral absorption coefficients $K_{\mathrm{e}}(\lambda)$ and $K_{\mathrm{d}}(\lambda)$ in the two layers are determined by the pigments in the following forms:

$$
\begin{aligned}
& K_{\mathrm{e}}(\lambda)=w_{m} K_{\mathrm{m}}(\lambda) \\
& K_{\mathrm{d}}(\lambda)=w_{h} K_{\mathrm{h}}(\lambda)+w_{d h} K_{\mathrm{dh}}(\lambda)
\end{aligned}
$$

where $K_{\mathrm{m}}(\lambda), K_{\mathrm{h}}(\lambda)$, and $K_{\mathrm{dh}}(\lambda)$ represent the spectral-absorption coefficients of melanin, oxy-hemoglobin, and deoxy-hemoglobin, respectively. The constant coefficients $w_{m}, w_{h}$, and $w_{d h}$ represent the weights of the pigment absorption coefficients.

For applying the estimation algorithm to real measurements, we need the optical and histological data of skin tissue. The spectral absorption data, the spectral scattering data, and the layer thickness values published in Refs [6,7] are used in this paper. 


\section{Procedure of Skin Color Simulation on Skin Surface}

The simulation is done by the estimation of spectral reflectance of skin surface with the change of pigment concentration and the display of the estimated color by the projection of the active illumination on the real skin surface. Fig. 4 shows the scheme of the skin color simulation. Spectral reflectances of skin surface with different concentrations of pigments are simulated. Then, the simulated colors are displayed on real skin surface by the projection of active illumination.

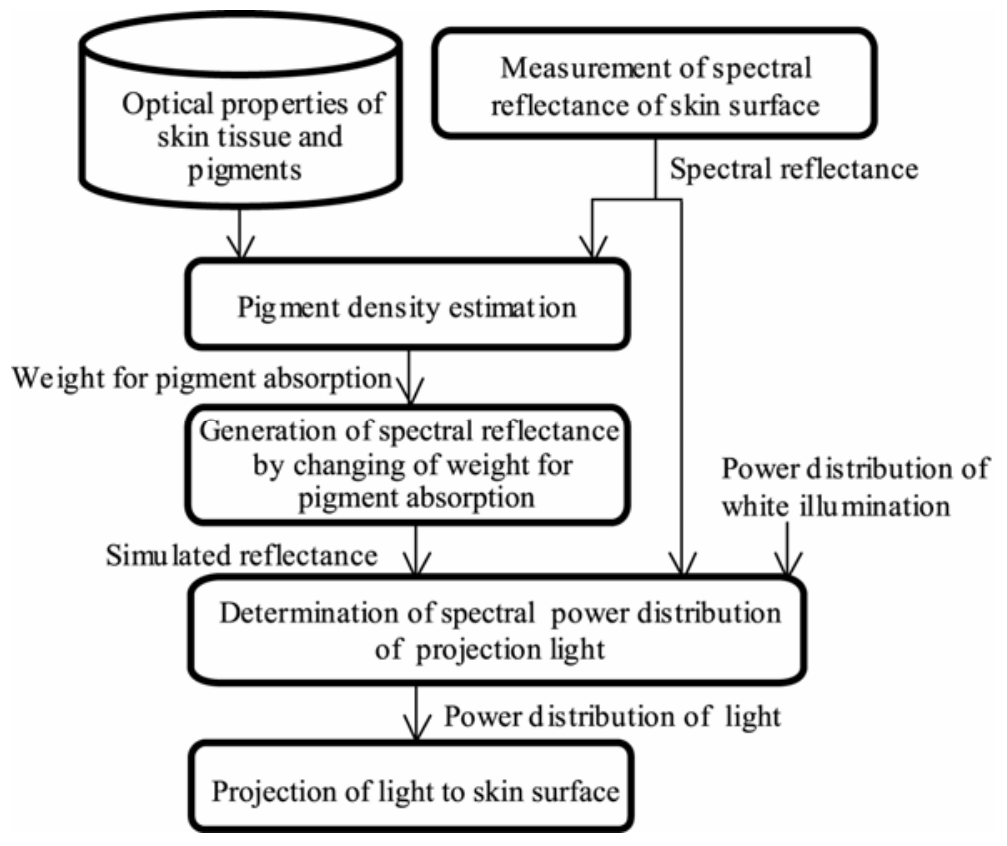

Fig. 4. Scheme of simulation procedure

\subsection{Simulation of Spectral Reflectance of Skin Surface with Condition Change}

The estimation of spectral reflectance of skin surface and the generation of new spectral reflectance of skin is done by the following procedures. First, the spectral reflectance of skin surface is measured. The measured skin surface area is the same area that is used for the display by the projection of the active illumination. Then, the fitting of the estimation data to the measured data is done by the control of the variables of pigment density in equation (7).

Next, new spectral reflectance data are generated by the changing of pigment density variables. When the weight for melanin is increased, the generated skin color shows the suntanned skin. The increase of hemoglobin generates reddish skin color by inflammation. 


\subsection{Display of Simulated Color on Real Skin Surface by Projection of Active Illumination}

The spectral power distributions of illuminations for simulated colors are calculated from the measured original spectral reflectance, the estimated spectral reflectances and the spectral power distribution of white illumination by equation (2). The spectral power distributions are converted to inputs of the programmable light source. The output of the programmable light source is calibrated in advance.

Then, the synthesized illumination is projected to the measured skin surface area in a room without other light sources. Simulated color appears on real skin surface under the synthesized illumination.

\section{Experiments}

We simulated melanin-increased skin color and hemoglobin-increased skin color on a back of hand. The programmable light source was adjusted beforehand. First, the spectral reflectance of skin was measured by a spectrometer. Fig. 5 shows the image of target skin and the spectral reflectance measured from the skin. The wavelengths of

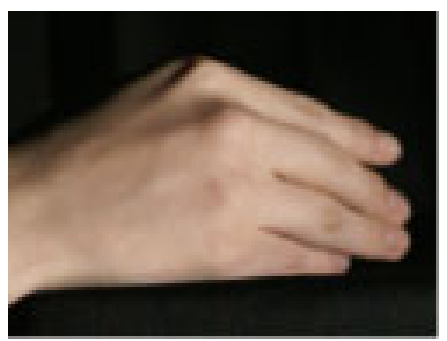

(a) Measurement target: back of hand

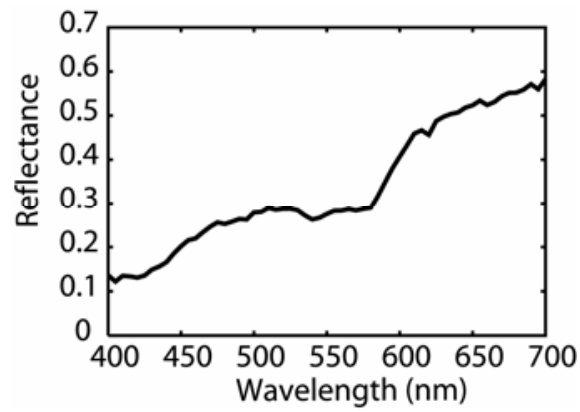

(b) Spectral reflectance of target

Fig. 5. Measurement of spectral skin reflectance

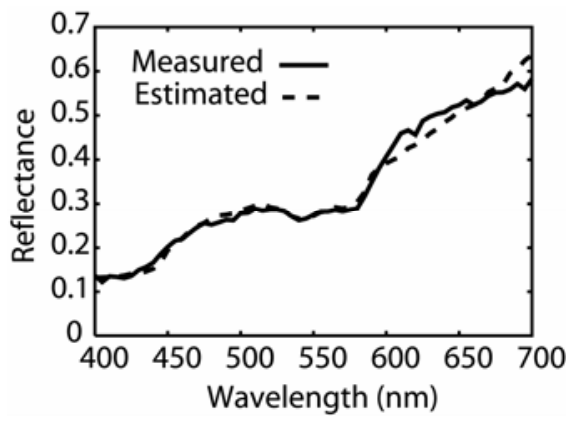

Fig. 6. Measured reflectance and fitted estimated reflectance 
Table 1. Weights for pigment absorption coefficients

\begin{tabular}{|l|c|}
\hline Pigment & Density coefficient \\
\hline Melanin $\left(w_{m}\right)$ & 470 \\
\hline Oxy-hemoglobin $\left(w_{h}\right)$ & 152 \\
\hline Deoxy-hemoglobin $\left(w_{d h}\right)$ & 200 \\
\hline
\end{tabular}

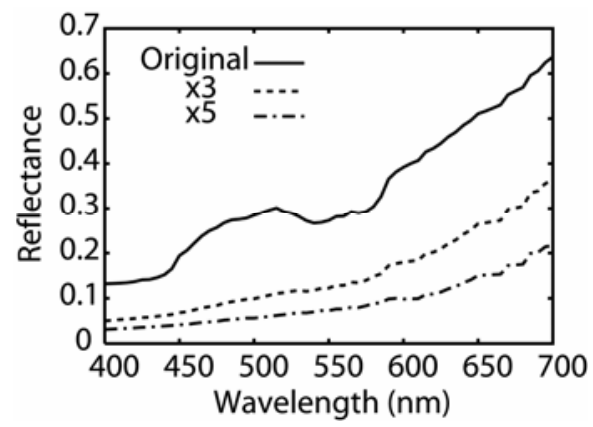

(a) Melanin-increased skin

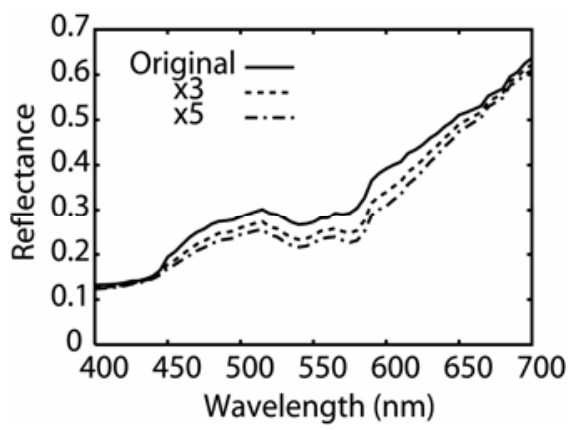

(b) Hemoglobin-increased skin

Fig. 7. Simulated spectral reflectance

the measured spectral reflectance were from $400 \mathrm{~nm}$ to $700 \mathrm{~nm}$ with the interval of $5 \mathrm{~nm}$. The weights of the pigment absorption coefficients were estimated from the measured spectral reflectance by the fitting of estimation data to the measured data. Figure 6 shows the measured data and the fitted estimation data. Table 1 shows the estimated weight parameters. Second, the spectral reflectances of skin with pigment density changes were simulated. In this experiment, the weights of pigment absorption coefficients were increased by a factor of three and five. Fig. 7 shows the spectral reflectances of original skin, simulated melanin-increased skin and simulated hemoglobin-increased skin. 
Next, the spectral power distributions of illuminations for the simulated colors were calculated from the measured original spectral reflectance, the estimated spectral reflectances and the spectral power distribution of white illumination by equation (2). Fig. 8 (a) shows the spectral power distribution of white illumination as $E(\lambda)$. One of the calculated spectral power distributions of illuminations for a simulated color, $E^{\prime}(\lambda)$, is shown in Fig. 8 (b).

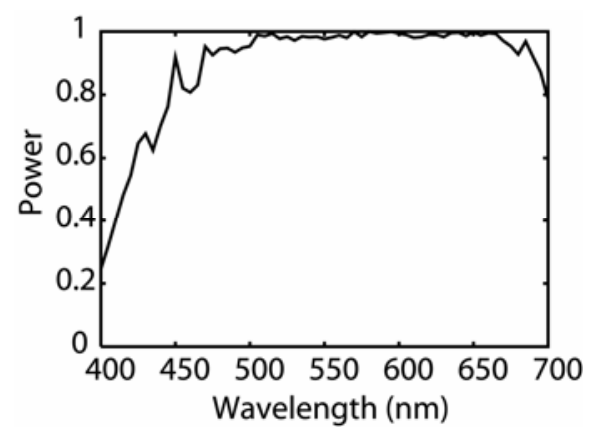

(a) White illumination

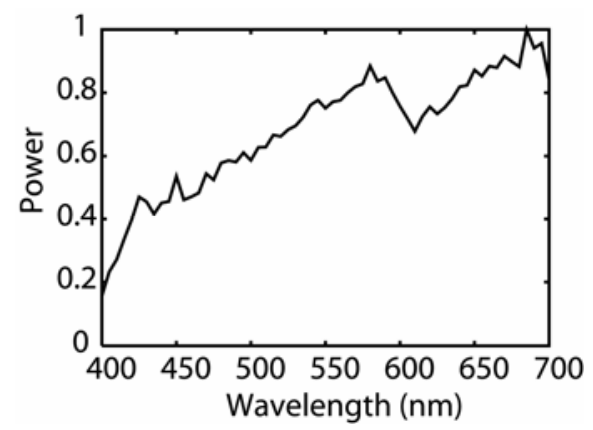

(b) Illumination for simulation of melanin-increased skin

Fig. 8. Spectral power distributions of illuminations of white and simulated color

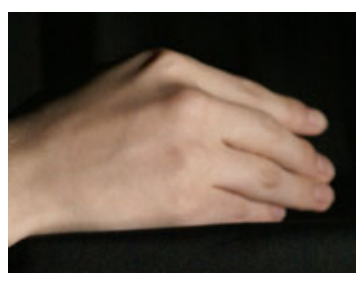

(a) Original

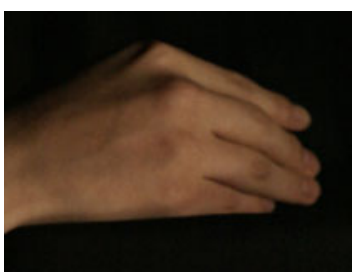

(b) Melanin x3

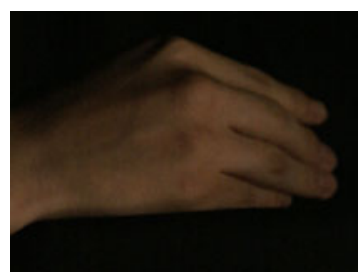

(c) Melanin x5

Fig. 9. Results of simulation for melanin-increased skin color 


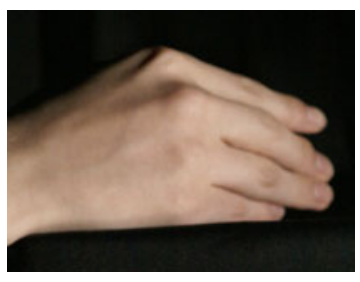

(a) Original

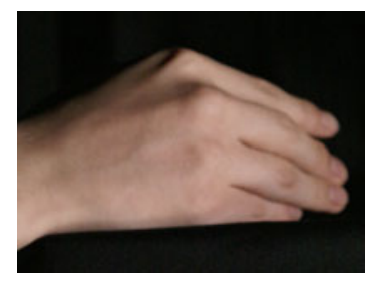

(b) Hemoglobin $\mathrm{x} 3$

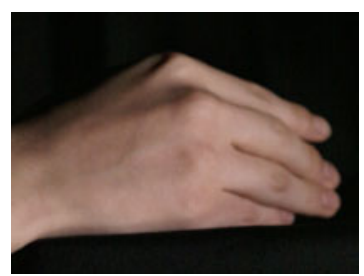

(c) Hemoglobin $x 5$

Fig. 10. Results of simulation for hemoglobin-increased skin color

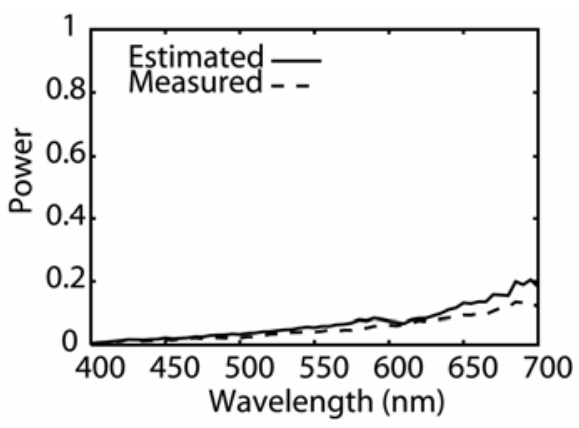

(a) Melanin

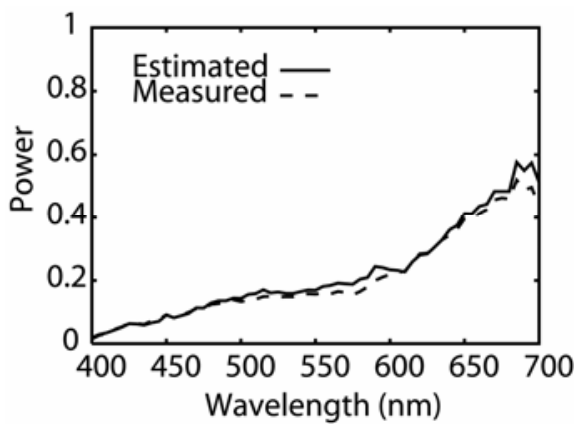

(b) Hemoglobin

Fig. 11. Comparison between estimated and measured spectral power distribution of light reflected on skin surface

Then, the colors of hand under these illuminations were observed. Fig. 9 shows pictures of hand with (a) original color, (b) simulated melanin-increased color by a factor of three and (c) simulated melanin-increased color by a factor of five. Fig. 10 shows pictures of hand with (a) original color, (b) simulated hemoglobin-increased color by a factor of three and (c) simulated hemoglobin-increased color by a factor of 
five. These pictures were taken by a digital camera with fixed camera parameters. These pictures show the feasibility of the proposed method.

Fig. 11 shows the comparison between the estimated spectral power distribution of reflected light on skin surface and measured spectral power distribution. Fig. 11 (a) shows the case of melanin-increased skin and Fig. 11 (b) shows the case of hemoglobin-increased skin. Average of absolute differences between the estimated data and measured data was 0.02 for each case.

\section{Conclusions}

This paper described the skin color simulation that displays simulated colors as spectral power distribution on real skin surface. The reproduction of the simulated color on skin is done by the illumination with the spectral power distribution calculated from the measured original spectral reflectance, the simulated spectral reflectances and the spectral power distribution of white illumination. We use a programmable light source to synthesize illuminant with arbitrary spectral-power distribution. We simulated skin color change by the concentration of pigments in skin. We estimated the spectral reflectances of melanin-increased skin and hemoglobin-increased skin by the method based on the Kubelka-Munk theory. Then, the estimated colors are displayed by the projection of the synthesized light on the real skin surface. The experimental results showed the feasibility of the proposed method.

\section{References}

1. Doi, M., Tanaka, N., Tominaga, S.: Spectral Reflectance Estimation of Human Skin and its Application to Image Rendering. J. Image Science and Technology 49(6), 574-582 (2005)

2. Kubelka, P.: New Contributions to the Optics of Intensely Light-Scattering Materials. Part I. J. Optical Society of America 38(5), 448-457 (1948)

3. Kubelka, P.: New Contributions to the Optics of Intensely Light-Scattering Materials. Part II. J. Optical Society of America 44(4), 330-335 (1954)

4. Tominaga, S., Horiuchi, T., Kakinuma, H., Kimachi, A.: Spectral Imaging with a Programmable Light Source. In: IS\&T/SID's 17th Color Imaging Conference, pp. 133-138 (2009)

5. Horiuchi, T., Kakinuma, H., Tominaga, S.: Effective Illumination Control for an Active Spectral Imaging System. In: 12th International Symposium on Multispectral Color Science (2010)

6. Anderson, R.R., Parrish, J.A.: The Optics of Human Skin. J. Investigative Dermatology 77(1), 13-19 (1981)

7. Gemert, M.J.C.V., Jacques, S.L., Sterenborg, H.J.C.M., Star, W.M.: Skin Optics. IEEE Transactions on Biomedical Engineering 36(12), 1146-1154 (1989) 